\title{
Application of support vector machines in detecting hand grasp gestures using a commercially off the shelf wireless myoelectric armband
}

\author{
Farshid Amirabdollahian ${ }^{1}$ and Michael L. Walters ${ }^{1}$
}

\begin{abstract}
The propose of this study was to assess the feasibility of using support vector machines in analysing myoelectric signals acquired using an off the shelf device, the Myo armband from Thalmic Lab, when performing hand grasp gestures.

Participants $(n=26)$ took part in the study wearing the armband and producing a series of required gestures. Support vector machines were used to train a model using participant training values, and to classify gestures produced by the same participants. Different Kernel functions and electrode combinations were studied. Also we contrasted different lengths of training values versus different lengths for the classification samples. The overall accuracy was $94.9 \%$ with data from 8 electrodes, and $72 \%$ where only four of the electrodes were used. The linear kernel outperformed the polynomial, and radial basis function. Exploring the number of training samples versus the achieved classification accuracy, results identified acceptable accuracies $(>90 \%)$ for training around $2.5 \mathrm{~s}$, and recognising grasp with $0.2 \mathrm{~s}$ of acquired data.

The best recognised grasp was the hand closed $(97.6 \%)$, followed by cylindrical grasp (96.8\%), the lateral grasp $(93.2 \%)$ and tripod (92\%). These results allows us to progress to the next stage of work where the Myo armband is used in the context of robot-mediated stroke rehabilitation and also involves more dynamic interactions as well as gross upper arm movements.
\end{abstract}

\section{INTRODUCTION}

The problem of detecting hand posture has been approached using various methods such as vision-based and glove-based approaches. Vision based approaches often involve detecting the fingertips and inferring joint-articulations using inverse kinematic models of the hand and the wrist skeleton [1]. Glove based approaches reduce the computation time by having a more-direct measurement of the articulations. Our earlier work using an electromechanical glove, the SCRIPT device, showed promising results in detecting pinch, lateral and cylindrical grasps. The glove measured the movements of hand and wrist which was fed to developed machine learning algorithms using Support Vector Machines (SVM), that achieved a detection accuracy of around $91 \%$ in identifying the type of grasp performed. The methods held for identifying gestures for people recovering from neurological conditions such as stroke. [2], [3]

Another possible approach is to utilise myoelectric signals recorded from arm and hand muscles in detecting gestures. Tavakolan et al. used SVM for pattern classification of surface electromyography signals of four forearm muscles in

\footnotetext{
*The authors acknowledge support from the EPSRC Centre for Innovative Manufacturing in Intelligent Automation, in undertaking this research work under grant reference number EP/IO33467/1

${ }^{1}$ Authors are with the Adaptive Systems Research Group, School of Computer Science, The University of Hertfordshire, Hatfield, United Kingdom. Contact author: f.amirabdollahian2@herts.ac.uk
}

order to classify eight hand gestures. They concluded that it was feasible to identify gestures using the four locally placed electrodes [4]. Similarly, Wang et al. used linear discriminant analysis to achieve an average accuracy of around $98 \%$ in detecting 8 hand gestures using two electrodes placed on the forearm [5]. Our study focuses on assessing the feasibility of using a commercially off the self device, the Myo armband from Thalmic labs, in detecting a number of hand grasp gestures using machine learning algorithm, particularly the support vector machines.

\section{MATERIAL AND METHODS}

A variety of biomedical and biomechanical assessments benefit from machine learning techniques. In a pioneering work, Doerschuk et. al explored using EMG signals to control a upper limb prosthetic arm and wrist [6]. Huang and Cheng extended the work into identifying a number of hand gestures [7]. Oskoei et. al assessed the use of support vector machines in order to identify the optimal feature set and kernels. Their study provided a comprehensive review of the techniques used and different available feature sets. [8]

In our earlier work, we utilised Support Vector Machines (SVM) in order to automatically and quickly identify a grasp intention. Participants in the study worn a robotic glove which was used to record the motion of their hand and wrist, and their sensed motion was used in training and classification of intended gestures. [3]. Our study showed acceptable accuracy of around $91 \%$ in detecting four grasps, tripod, lateral, cylindrical and rest gestures.

Our electromechanical device was subject to sensor drift and hence, in the current study, we aimed at applying the same technique to a different type of sensory data obtained using electromyography retrieved using the Myo armband from Thalmic $\mathrm{Lab}^{1}$. The armband is depicted in Fig 1. It consists of 8 proprietary Electromyography (EMG) electrodes placed equidistally around the arm utilising an ARM Cortex M4 processor to communicate via Bluetooth 4. The device offers position tracking using accelerometers, gyroscope and magnetometers, and also haptic feedback in form of vibration. Unlike earlier studies where individual electrodes are applied to flexor and extensor muscles, the Myo armband offers the possibility of positioning the electrodes at a relatively fixed location with respect to one another. Electrode application is simple and the device can be worn at home without assistance. We used the Robot

${ }^{1}$ https://www.thalmic.com/en/myo/ 


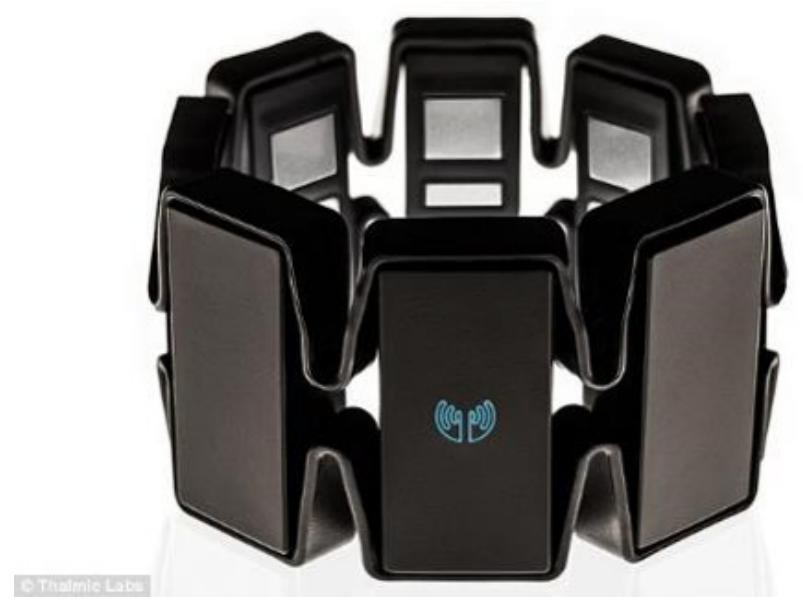

Fig. 1. Myo armband from Thalmic Labs

Operating System ${ }^{2}$ to develop an application that acquired data from individual electrodes which would allow for future testing of the interface with robots.

\section{A. Experiment Design}

The designed experiment had three phases. During phase A, participants made themselves familiar with the armband and its operation. During this time, participants tried 4 gestures that are currently detected by the device software. As these gestures were performed by each participant, the relevant image showing the hand in its recognised gesture appeared on screen. Familiarisation gestures were closed fist, hand open with fingers spread, wrist fully flexed and wrist fully extended as depicted in Fig 2. When participants were confident in using the device, they then moved to the next phase.

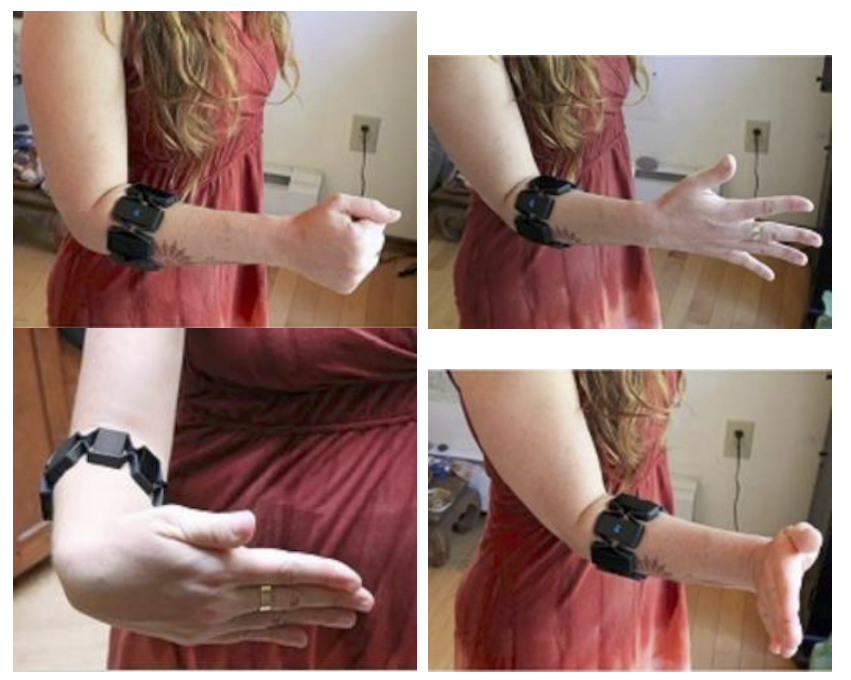

Fig. 2. Gestures used for familiarisation with Myo. Left to right: Closed fist, fingers spread, wrist flexed and wrist extended

${ }^{2}$ www.ros.org
In phase $\mathrm{B}$, the training phase, participants tried one of the four gestures shown in Figure 3 that were presented in a random order on screen. Each image was presented for 5 seconds, and electrode readings logged at $50 \mathrm{~Hz}$. During this 5 seconds, the participant was told to hold the hand in the same grasp posture as presented on screen. Once all of the four gestures were performed 5 times, participants moved to the next phase of the study. This allowed capturing 25 seconds of data for each of the gestures in Figure 3.
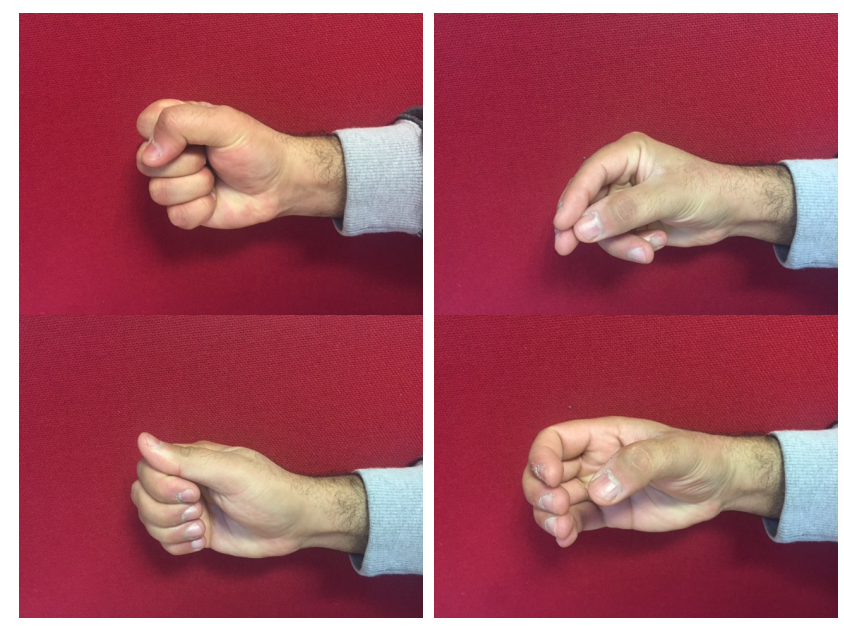

Fig. 3. Left to right: fist, tripod, lateral and cylindrical grasps randomly presented during training phase B

In phase $\mathrm{C}$, or the automatic recognition phase, the same gestures used in Phase B are shown on screen. This time the produced gesture is recognised using the a machine learning algorithm (detailed in [9]) and the resulting gesture code is labelled as $\{0,1,2,3\}$ corresponding to closed fist, tripod, lateral and cylindrical grasps. Labels are logged alongside the presented gesture codes at $50 \mathrm{~Hz}$. Overall, considering the three phases, a typical experiment session is shorter than 15 minutes.

For this study, only the data obtained during phase B is used to derive the machine learning models and to validate the model derivation.

\section{B. Participants and Experiment setup}

The experiment protocol was approved by the University of Hertfordshire's ethics committee under the approval number COM/PGR/UH/02057. A total of 26 participants consented to take part in the study. Participants sat in front of a 21 inch monitor, wearing the Myo armband on their dominant arm. The forearm was rested on a Saebo MAS arm support to limit additional muscle contractions. The experimental setup is offered in Fig 5.

During the experiment, due to technical issues, one participant did not complete the study. All remaining participants $(n=25)$ completed the three phases of the study. The participant aged varied from 24 to 59 years $(M=38, S D=10)$, with 9 female participants and 16 males. Twenty participants were right handed while the remaining 3 were left handed. 


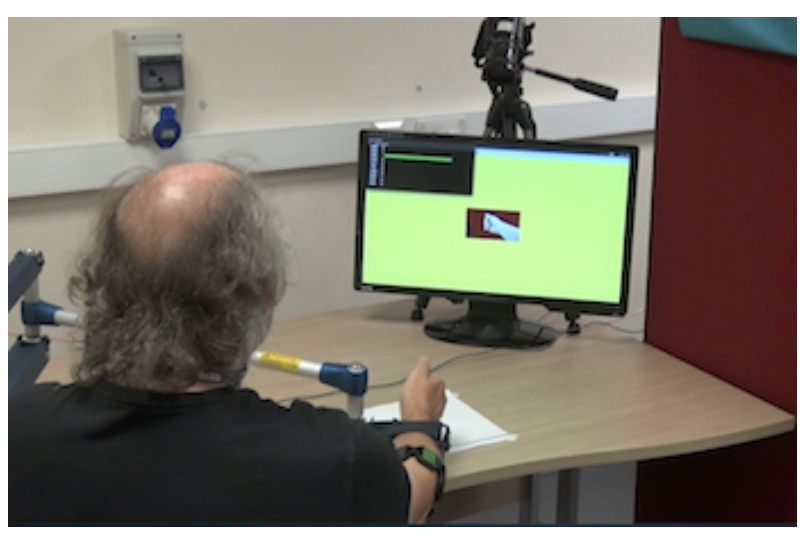

Fig. 4. Experimental setup

Participants 24, 21, 18 and 13 had a medical history of previous injury to their hand and wrist.

\section{Methodology}

Our earlier study with SCRIPT device showed promising results for using machine learning in identifying gestures with an electromechanical glove. In the followup work, we continued to assess the utility of multiple approaches in machine learning in detecting gestures using myoelectric signals. The study of k-nearest neighbour method in classifying myoelectric signals resulted in [9]. The current paper focuses on utility of SVM in gesture classification. To do so, it utilises the data recorded during the second phase of the study where participants repeated performed random gestures for a duration of 5 seconds, 5 times.

Support vector machines are increasingly popular tools for machine learning. The support vectors are constructed optimal hyperplanes in a multidimensional feature space. They allow for clustering and separation of the data classes, but also allow to assign class labels to new observations. The theoretical background and how these machines work in further details is offered in [10] and its application in analysing human-robot sensed interaction is presented by Leon et. al [2], and the application in assessing electromygraphy data is detailed by Oskoei and $\mathrm{Hu}$ [8]. Both application examples utilise the libsvm, a powerful library for support vector machines [11] which has been also used for the analysis presented in this paper.

For the data preparation in this paper, the recorded data obtained from phase B of the experiment, the familiarisation phase, is divided into two parts, one part is used for training while the second part is used to test and validate the trained machine. We have experimented with different training/classification segment lengths in order to identify the best classification accuracies. We derived the training and classification sets for each participant by considering different lengths for the training training $s \in\{2.5,3.0,3.5,4.0\}$ seconds and also for different lengths for the classification seconds as classification_s $\in\{0.2,0.4,0.6,0.8,1.0\}$.

Raw data recorded from the EMG sensors in the Myo armband are recorded as an array of 8 values with a $50 \mathrm{~Hz}$ sampling rate. Before the training, we had to identify a potent feature set or objective representation. Oskoei and $\mathrm{Hu}$ showed that the waveform length (WL) feature is capable of detecting gestures with an acceptable and robust performance [8]. Huang and Chen [7] offer the waveform length as:

$$
W L=\sum_{k=1}^{n} \quad\left|x_{k}-x_{k-1}\right|
$$

Noting that the minimum interval between two distinct muscle contraction is approximately $200 \mathrm{~ms}$ [12] and that this segment length had been found to be a suitable length for a SVM application [8], we used $n=10$ in equation (1) to reduce the data to $200 \mathrm{~ms}$ interval features which are then used for machine learning and classification. Based on the lengths for training_s and classification_s sets, these resulted in a number of training observations for different pairs of training and classification durations. For example, in case of a pair (training_s $=2.5$, classification_s $=0.2$ ), 125 training samples are reduced to $12 \mathrm{WL}$ values and used to train the SVM. On the classification side, $200 \mathrm{~ms}$ data captured in 10 samples are reduced to one WL value. Given the 5 repeated grasp gestures by each participant, this provides 60 training samples and 5 classification WL data points.

We used python libsvm libraries and passed the training data through an initial assessment (easy.py from the libsvm tools) to identify the kernel parameters for three different kernels: linear, polynomial and RBF kernel. Each problem set consisting of a training and classification set are treated to identify their relevant training parameters. In the case of an RBF kernel, a pair of $(c, \gamma)$ training parameters were identified. For the polynomial and linear kernels, the optimal parameter $c$ was identified. Using these, the libsvm libraries calculate recognition accuracy by comparing predicted labels to expected labels for each individual participant and each of the samples in the classification set. The resulting recognition accuracies are then gathered in a $C S V$ file and analysed using IBM SPSS version 23 for calculating descriptive statistics.

\section{RESULTS}

We experimented with 3 different kernels as offered in table I. The table shows the differences in overall recognition accuracy for the same amount of training data used for each kernel:

TABLE I

TRAINING ACCURACY FOR DIFFERENT SVM KERNELS

\begin{tabular}{|c|c|c|}
\hline Kernel & Accuracy (mean) & Std. Deviation \\
\hline Linear & 94.90 & 11.13 \\
Polynomial & 89.80 & 14.71 \\
Radial Basis Function & 31.10 & 20.48 \\
\hline
\end{tabular}

This identified the linear kernel as the most accurate kernel to be used for the training and recognition. A similar trend was visible when comparing the kernel performance between different grasps. 
The next comparison explores accuracy gains based on different training and recognition sample lengths as offered by pairing choices in training_s and recognition_s.

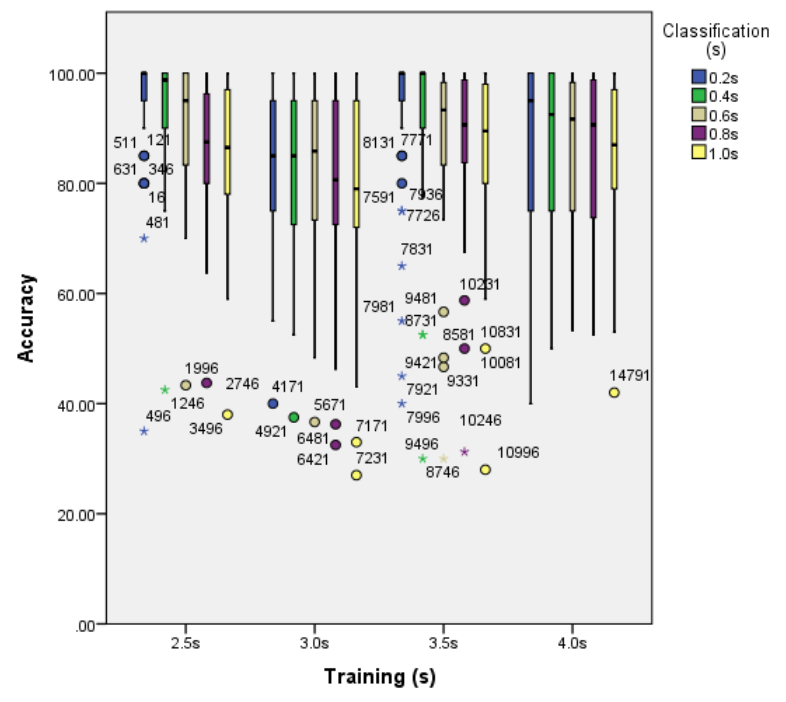

Fig. 5. Recognition accuracies for different training and recognition lengths

This identified the pair where training $s=2.5$ and classification_s $=0.2$ as the most accurate $(94.9 \pm 11.13)$ lengths for this classification problem. Table II shows the accuracy for different gestures with this optimal training and classification lengths:

TABLE II

TRAINING ACCURACY FOR DIFFERENT GRASPS IN THIS STUDY OBTAINED USING 8 ELECTRODES

\begin{tabular}{|c|c|c|}
\hline Grasp & Mean & Std. Deviation \\
\hline Fist & 97.60 & 9.59 \\
Cylindrical & 96.80 & 11.68 \\
Lateral & 93.20 & 19.63 \\
Tripod & 92.00 & 22.13 \\
\hline
\end{tabular}

Fig 6 presents the accuracy of grasp recognition for different participants in this study, performing different gestures.

Finally, the Myo armband utilises 8 electrodes placed around the arm. We explored whether a similar recognition can be achieved if only 4 of these electrodes were used, using only every other electrode around the arm. We used training_s $=2.5 \mathrm{~s}$ and classification_s $=0.2 \mathrm{~s}$ and the linear kernel. This resulted in a overall classification accuracy of $72.5 \pm 17.2$ percent and also lower recognition accuracies for the individual grasps as provided in Table III.

\section{DISCUSSIONS \& CONCLUSIONS}

This study compared the classification accuracy of detecting gestures using myoelectric signals acquired from the commercially off the shelf device, Myo armband. We compared classifications between different kernels and concluded that the linear kernel out performed the polynomial and RBF kernels. This is different to the results offered

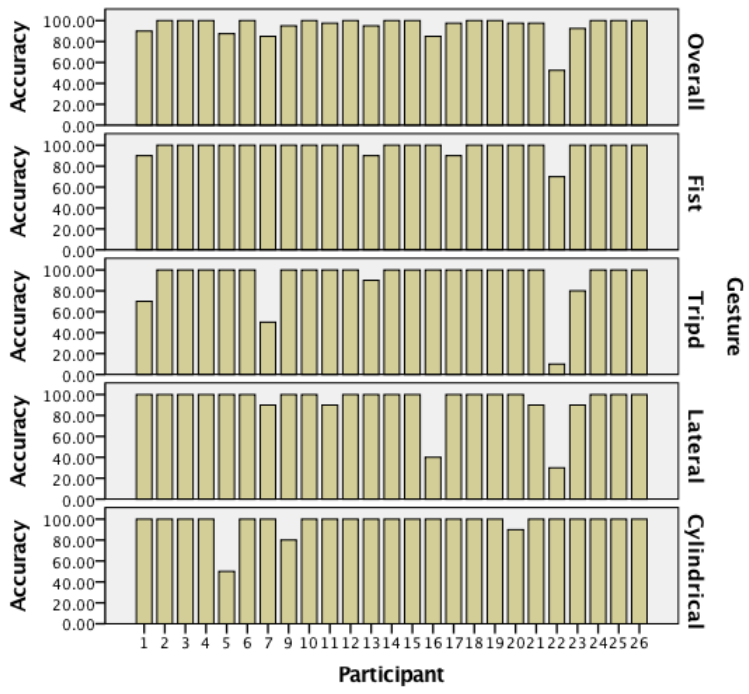

Fig. 6. Classification accuracies for different gestures performed by participants

TABLE III

TRAINING ACCURACY FOR DIFFERENT GRASPS IN THIS STUDY OBTAINED USING 4 ELECTRODES

\begin{tabular}{|c|c|c|}
\hline Grasp & Mean & Std. Deviation \\
\hline Fist & 68.16 & 31.29 \\
Cylindrical & 77.33 & 35.91 \\
Lateral & 64.41 & 19.63 \\
Tripod & 80.25 & 28.05 \\
\hline
\end{tabular}

by Oskoei and $\mathrm{Hu}$ [8] showing similar performances across these kernels. Our study used a significantly lower sampling frequency $(50 \mathrm{~Hz}$ compared to a $1000 \mathrm{~Hz}$ in the cited study) and therefore had a smaller training dataset. In our case, the RBF kernel often did not identify the optimal training parameters given the number of features and provided number of observations. Also our study acquired the signals from muscles located at a cross-section of the arm covered by the armband while Oskoei and $\mathrm{Hu}$ acquired the signals from electrodes placed at different locations along the length of the arm. In our next study we will take full advantage of the whole $200 \mathrm{~Hz}$ sampling rate available to compare if different kernel performances would be improved given this.

We applied the waveform length to compare classification accuracy for different amounts of training and classification data. Our results indicated that a $2.5 \mathrm{~s}$ training data repeated 5 times is capable of returning acceptable accuracies for $0.2 \mathrm{~s}$ of the classification data from the same gesture/grasp. Expectedly, longer classification data has larger deviation and bigger loss in accuracy. An interesting observation here is that classification results for $2.5 \mathrm{~s}$ and $3.5 \mathrm{~s}$ of the training data are similar, while classification results for $3.0 \mathrm{~s}$ and 4.0s of training data are also similar to one another (see Fig 5). We hypothesise that this results from a chance assignment of training length iterations (increase by 0.5 seconds per problem) that may better contain a complete 
waveform length related to the full grasps. Participants in this study produced the training grasps using the required grasp's image on screen and it could be argued that different grasp speeds as well as perception times needed to produce the grasps may result in different accuracies observed. To ascertain this, we currently plan a study that provides the required grasp in an animated form on screen accompanied by audio prompts, thus to ensure all participants produce the exact gestures in a given time, and also to explore further the link between grasp accuracy similarities observed here. This will also allow us to shed further light into cases where grasp classification accuracy is significantly lower, for example in the case of participant 22 where all gestures but the cylindrical grasp show lower classification accuracy. In this case, the optimisation process estimating the pair of $(c, \gamma)$ parameters did not manage to reach an optimal solution given the extent of training data offered. Further exploration in this area will allow us to identify if Myo arm band offers a reliable source of data for all participants, and whether additional training data can offer better classification accuracies in such cases.

We also repeated our study while only using 4 of the 8 electrodes available. Our results indicated a drop in classification accuracy, that does not match the classification accuracies reported by [4]. We anticipate that this could also have been influenced by our slow sampling rate and also the location of the electrodes around the arm. A follow-up study with faster sampling frequency is in progress to allow for accounting for the sampling rate effects.

This study presented reasonable accuracies for the grasps examined enabling us to move to the next phases where these grasps can be automatically detected using the trained support vectors, thus enabling us to provide a more accurate interactive rehabilitation exercise. Our future studies will aim at reducing the observed variations shown in Figure 6 thus to make the interaction more reliable. In this study, we supported the weight of the arm using an arm support device, which could reduce the variability of EMG signals acquired from the lower arm. We currently have an ongoing followup study where larger unsupported arm motions are assessed dynamically to predict their intentions.

Furthermore we acknowledge that this study has performed the analysis with healthy individuals in a laboratory setting, while intended target users are people recovering from stroke. Our findings will be re-examined in a similar experiment with the intended users in future studies.

Lastly, we aimed at identifying if a commercially available off-the-shelf device would provide sufficient data for the machine learning models. We confirm this with a our limited $50 \mathrm{~Hz}$ sampling assignment, a small amount of training data that can be captured in a calibration phase or personalisation phase of a human-robot interaction session. Our next planned study will compare this performance to a medical TMSI amplifier with clinical EMG.

The current experiment gather data from a short-time use of the device and a remaining question relates to repeatability of these results after a longer exposure and use in different days and different times of the day. These remain a motivation for our future studies.

\section{ACKNOWLEDGMENT}

The authors acknowledge support from the EPSRC Centre for Innovative Manufacturing in Intelligent Automation, in undertaking this research work under grant reference number EP/IO33467/1. The authors also thank the participants in this study and Mr Rory Heffernan for data collection.

\section{REFERENCES}

[1] A. Chaudhary, J. L. Raheja, K. Das, and S. Raheja, "Intelligent approaches to interact with machines using hand gesture recognition in natural way: a survey," arXiv preprint arXiv:1303.2292, 2013.

[2] B. Leon, A. Basteris, and F. Amirabdollahian, "Comparing recognition methods to identify different types of grasp for hand rehabilitation," in 7th International Conference on Advances in Computer-Human Interactions.(ACHI2014), 2014, pp. 109-114.

[3] B. Leon, A. Basteris, F. Infarinato, P. Sale, S. Nijenhuis, G. Prange, and F. Amirabdollahian, "Grasps recognition and evaluation of stroke patients for supporting rehabilitation therapy," BioMed Research International, vol. 2014, 2014.

[4] M. Tavakolan, Z. G. Xiao, and C. Menon, "A preliminary investigation assessing the viability of classifying hand postures in seniors," Biomedical engineering online, vol. 10, no. 1, p. 1, 2011.

[5] N. Wang, Y. Chen, and X. Zhang, "The recognition of multi-finger prehensile postures using lda," Biomedical Signal Processing and Control, vol. 8, no. 6, pp. 706-712, 2013.

[6] P. C. Doerschuk, D. E. Gustafon, and A. S. Willsky, "Upper extremity limb function discrimination using emg signal analysis," IEEE Transactions on Biomedical Engineering, no. 1, pp. 18-29, 1983.

[7] H.-P. Huang and C.-Y. Chen, "Development of a myoelectric discrimination system for a multi-degree prosthetic hand," in Robotics and Automation, 1999. Proceedings. 1999 IEEE International Conference on, vol. 3. IEEE, 1999, pp. 2392-2397.

[8] M. A. Oskoei and H. Hu, "Support vector machine-based classification scheme for myoelectric control applied to upper limb," IEEE transactions on biomedical engineering, vol. 55, no. 8, pp. 1956-1965, 2008.

[9] F. Amirabdollahian, M. Walters, R. Heffernan, S. Fletcher, and P. Webb, "Using myoelectric signals for gesture detection: a feasibility study," in Ergonomics and Human Factors. Chartered Institute of Ergonomics and Human Factors, 2017.

[10] C. Cortes and V. Vapnik, "Support-vector networks," Machine learning, vol. 20, no. 3, pp. 273-297, 1995.

[11] C.-C. Chang and C.-J. Lin, "LIBSVM: A library for support vector machines," ACM Transactions on Intelligent Systems and Technology, vol. 2, pp. 27:1-27:27, 2011, software available at http://www.csie.ntu.edu.tw/ cjlin/libsvm.

[12] J. Valls-Solé, J. C. Rothwell, F. Goulart, G. Cossu, and E. Munoz, "Patterned ballistic movements triggered by a startle in healthy humans," The Journal of physiology, vol. 516, no. 3, pp. 931-938, 1999. 